\title{
Sub-super solutions for $(p-q)$ Laplacian systems
}

\author{
Somayeh Haghaieghi ${ }^{1 *}$ and Ghasem Alizadeh Afrouzi ${ }^{2}$
}

* Correspondence:

Haghaieghi_ch86@yahoo.com 'Department of Mathematics, Science and Research Branch, Islamic Azad University, Tehran, Iran Full list of author information is available at the end of the article

\section{Abstract}

In this work, we consider the system:

$$
\begin{cases}-\Delta_{p} u=\lambda[g(x) a(u)+f(v)] & \text { in } \Omega \\ -\Delta_{q} v=\lambda[g(x) b(v)+h(u)] & \text { in } \Omega \\ u=v=0 & \text { on } \partial \Omega,\end{cases}
$$

where $\Omega$ is a bounded region in $R^{N}$ with smooth boundary $\partial \Omega, \Delta_{p}$ is the p-Laplacian operator defined by $\Delta_{p} u=\operatorname{div}\left(|\nabla u|^{p-2} \nabla u\right), p, q>1$ and $g(x)$ is a $C^{1}$ sign-changing the weight function, that maybe negative near the boundary. $f, h, a, b$ are $C^{1}$ nondecreasing functions satisfying $a(0) \geq 0, b(0) \geq 0$. Using the method of sub-super solutions, we prove the existence of weak solution.

\section{Content}

In this paper, we study the existence of positive weak solution for the following system:

$$
\left\{\begin{array}{cc}
-\Delta_{p} u=\lambda[g(x) a(u)+f(v)] & \text { in } \Omega \\
-\Delta_{q} v=\lambda[g(x) b(v)+h(u)] & \text { in } \Omega \\
u=v=0 & \text { on } \partial \Omega,
\end{array}\right.
$$

where $\Omega$ is a bounded region in $R^{N}$ with smooth boundary $\partial \Omega, \Delta_{p}$ is the p-Laplacian operator defined by $\Delta_{p} u=\operatorname{div}\left(|\nabla u|^{p-2} \nabla u\right), p, q>1$ and $g(x)$ is a $C^{1}$ sign-changing the weight function, that maybe negative near the boundary. $f, h, a, b$ are $C^{1}$ non-decreasing functions satisfying $a(0) \geq 0, b(0) \geq 0$.

This paper is motivated by results in [1-5]. We shall show the system (1) with signchanging weight functions has at least one solution.

\section{Preliminaries}

In this article, we use the following hypotheses:

(Al)

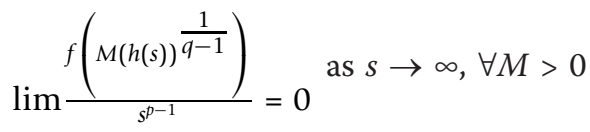

(A2) $\lim f(s)=\lim h(s)=\infty$ as $s \rightarrow \infty$.

(A3) $\lim \frac{a(s)}{s^{p-1}}=\lim \frac{b(s)}{s^{q-1}}=0$ as $s \rightarrow \infty$.

Let $\lambda_{p}, \lambda_{q}$ be the first eigenvalue of $-\Delta_{p},-\Delta_{q}$ with Dirichlet boundary conditions and $\phi_{p}, \phi_{q}$ be the corresponding positive eigenfunctions with $\left\|\phi_{p}\right\|_{\infty}=\left\|\phi_{q}\right\|_{\infty}=1$. 
Let $m, \delta, \gamma, \mu_{p}, \mu_{q}>0$ be such that

$$
\left\{\begin{aligned}
\left|\nabla \varphi_{p}\right|^{p}-\lambda_{p} \varphi_{p} & \geq m \quad \text { in } \bar{\Omega}_{\delta} \\
\varphi_{p} & \geq \mu_{p} \quad \text { on } \Omega-\Omega_{\delta}
\end{aligned}\right.
$$

and

$$
\begin{aligned}
& \left\{\begin{array}{r}
\left|\nabla \varphi_{q}\right|^{q}-\lambda_{q} \varphi_{q} \geq m \quad \text { in } \bar{\Omega}_{\delta} \\
\varphi_{p} \geq \mu_{p} \text { on } \Omega-\Omega_{\delta} .
\end{array}\right. \\
& \bar{\Omega}_{\delta}=\{x \in \Omega ; d(x, \partial \Omega) \leq \delta\} .
\end{aligned}
$$

We assume that the weight function $g(x)$ take negative values in $\Omega_{\delta}$, but it requires to be strictly positive in $\Omega-\Omega_{\delta}$. To be precise, we assume that there exist positive constants $\beta$ and $\eta$ such that $g(x) \geq-\beta$ on $\bar{\Omega}_{\delta}$ and $g(x) \geq \eta$ on $\Omega-\Omega_{\delta}$. Let $s_{0} \geq 0$ such that $\eta a(s)+f(s)>0, \eta b(s)+h(s)>0$ for $s>s_{0}$ and

$$
f_{0}=\max \{0,-f(0)\}, h_{0}=\max \{0,-h(0)\} .
$$

For $\gamma$ such that $\gamma^{r-1} t>s_{0} ; t=\min \left\{\alpha_{p}, \alpha_{q}\right\}, r=\min \{p, q\}$ we define

$$
\begin{aligned}
& A=\max \left[\frac{\gamma \lambda_{p}}{\eta\left(\frac{1}{p-1} \alpha_{p}\right)+f\left(\frac{1}{q-1} \alpha_{q}\right)}, \frac{\gamma \lambda_{q}}{\eta b\left(\frac{1}{q-1}{ }_{\alpha_{q}}\right)+h\left(\frac{1}{p-1} \alpha_{\alpha_{p}}\right)}\right] \\
& \left.B=\min \left[\frac{m \gamma}{\beta a\left(\frac{1}{p-1}\right)+f_{0}}, \frac{m b\left(\frac{m}{1}\right)}{q-1}\right)+h_{0}\right]
\end{aligned}
$$

where $\alpha_{p}=\frac{p-1}{p} \mu_{p} \frac{p}{p-1}$ and $\alpha_{q}=\frac{q-1}{q} \mu_{q} \frac{q}{q-1}$.

We use the following lemma to prove our main results.

Lemma 1.1 [6]. Suppose there exist sub and supersolutions $\left(\psi_{1}, \psi_{2}\right)$ and $\left(z_{1}, z_{2}\right)$ respectively of (1) such that $\left(\psi_{1}, \psi_{2}\right) \leq\left(z_{1}, z_{2}\right)$. then (1) has a solution $(u, v)$ such that $(u, v) \in\left[\left(\psi_{1}, \psi_{2}\right),\left(z_{1}, z_{2}\right)\right]$.

\section{Main result}

Theorem 3.1Suppose that (A1)-(A3) hold, then for every $\lambda \in[A, B]$, system (1) has at least one positive solution.

Proof of Theorem 3.1 We shall verify that $\left(\psi_{1}, \psi_{2}\right)$ is a sub solution of (1.1) where

$$
\begin{aligned}
& \psi_{1}=\gamma^{\frac{1}{p-1}} \frac{p-1}{p} \varphi_{p} \frac{p}{p-1} \\
& \psi_{2}=\gamma^{\frac{1}{q-1}} \frac{q-1}{q} \varphi_{q} \frac{q}{q-1} .
\end{aligned}
$$


Let $W \in H_{0}{ }^{1}(\Omega)$ with $w \geq 0$. Then

$$
\int_{\Omega}\left|\nabla \psi_{1}\right|^{p-2} \nabla \psi_{1} \nabla w \mathrm{~d} x=\gamma \int_{\Omega}\left(\lambda_{p} \varphi_{p}^{p}-\left|\nabla \varphi_{p}\right|^{p}\right) w \mathrm{~d} x
$$

Now, on $\bar{\Omega}_{\delta}$ by (2),(3) we have

$$
\gamma\left(\lambda_{p} \varphi_{p}^{p}-\left|\nabla \varphi_{p}\right|^{p}\right) \leq-m \gamma
$$

Since $\lambda \leq B$ then

$$
\lambda \leq \frac{m \gamma}{\beta a\left(\gamma^{\frac{1}{p-1}}\right)+f_{0}} .
$$

thus

$$
\begin{aligned}
\gamma\left(\lambda_{p} \varphi_{p}^{p}-\left|\nabla \varphi_{p}\right|^{p}\right) & \leq-m \gamma \\
& \leq \lambda\left(-\beta a\left(\gamma^{\frac{1}{p-1}}\right)-f_{0}\right) \\
& \leq \lambda\left(g(x) a\left(\gamma^{\frac{1}{p-1}}\right)-f_{0}\right) \lambda\left(g(x) a\left(\frac{p-1}{p} \gamma^{\frac{1}{p-1}} \varphi_{p}^{\frac{1}{p-1}}\right)\right. \\
& \left.+f\left(\frac{q-1}{q} \gamma^{\frac{1}{q-1}} \varphi_{q}^{\frac{1}{q-1}}\right)\right)
\end{aligned}
$$

then by (4)

$$
\begin{aligned}
\int_{\overline{\Omega_{\delta}}}\left|\nabla \psi_{1}\right|^{p-2} \nabla \psi_{1} \nabla w \mathrm{~d} x \leq & \int_{\bar{\Omega}_{\delta}} \lambda\left(g(x) a\left(\frac{p-1}{p} \gamma^{\frac{1}{p-1}} \varphi_{p}^{\frac{p}{p-1}}\right)\right. \\
& \left.+f\left(\frac{q-1}{q} \gamma^{\frac{1}{q-1}} \varphi_{q}^{\frac{q}{q-1}}\right)\right) w \mathrm{~d} x
\end{aligned}
$$

A similar argument shows that

$$
\begin{aligned}
\int_{\bar{\Omega}_{\delta}}\left|\nabla \psi_{2}\right|^{q-2} \nabla \psi_{2} \nabla w \mathrm{~d} x \leq & \int_{\bar{\Omega}_{\delta}} \lambda\left(g(x) b\left(\frac{q-1}{q} \gamma^{\frac{1}{q-1}} \varphi_{q} \frac{1}{q-1}\right)\right. \\
& \left.+h\left(\frac{p-1}{p} \gamma^{\frac{1}{p-1}} \varphi_{p} \frac{1}{p-1}\right)\right) w \mathrm{~d} x
\end{aligned}
$$

Next, on $\Omega-\bar{\Omega}_{\delta}$. Since $\lambda \geq A$, then

$$
\lambda \geq \frac{\gamma \lambda_{p}}{\eta a\left(\gamma^{\frac{1}{p-1}} \alpha_{p}\right)+f\left(\gamma^{\frac{1}{q-1}} \alpha_{q}\right)}
$$

so we have

$$
\begin{aligned}
\gamma\left(\lambda_{p} \varphi_{p}^{p}-\left|\nabla \varphi_{p}\right|^{p}\right) & \leq \gamma \lambda_{p} \\
& \leq \lambda\left[\eta a\left(\gamma^{\frac{1}{p-1}} \alpha_{p}\right)+f\left(\gamma^{\frac{1}{q-1}} \alpha_{q}\right)\right] \\
& \leq \lambda\left[g(x) a\left(\psi_{1}\right)+f\left(\psi_{2}\right)\right], \Omega-\overline{\Omega_{\delta}}
\end{aligned}
$$


Then by (4) on we have

$$
-\Delta_{p} \psi_{1} \leq \lambda\left[g(x) a\left(\psi_{1}\right)+f\left(\psi_{2}\right)\right] \quad \text { on } \Omega-\overline{\Omega_{\delta}}
$$

A similar argument shows that

$$
-\Delta_{q} \psi_{2} \leq \lambda\left[g(x) b\left(\psi_{2}\right)+h\left(\psi_{1}\right)\right]
$$

We suppose that $\kappa_{p}$ and $\kappa_{q}$ be solutions of

$$
\begin{aligned}
& \left\{\begin{aligned}
-\Delta_{p} \kappa_{p}=1 & \text { in } \Omega \\
\kappa_{p}=0 & \text { on } \partial \Omega
\end{aligned}\right. \\
& \left\{\begin{aligned}
-\Delta_{q} \kappa_{q}=1 & \text { in } \Omega \\
\kappa_{q}=0 & \text { on } \partial \Omega
\end{aligned}\right.
\end{aligned}
$$

respectively, and $\mu_{p}^{\prime}=\left\|\kappa_{p}\right\|_{\kappa},\left\|\kappa_{q}\right\|_{\kappa}=\mu_{q}^{\prime}$.

Let

$$
\left(z_{1}, z_{2}\right)=\left(\frac{c}{\mu_{p}^{\prime}} \lambda^{\frac{1}{p-1}} \kappa_{p},\left[2 h\left(c \lambda^{\frac{1}{q-1}}\right)\right]^{\frac{1}{q-1}} \lambda^{\frac{1}{q-1}} \kappa_{q}\right)
$$

Let $W \in H_{0}{ }^{1}(\Omega)$ with $w \geq 0$.

For sufficient $C$ large

$$
\frac{\mu_{p}^{\prime p-1}\left[\|g\|_{\infty} a\left(C \lambda^{\frac{1}{p-1}}\right)+f\left(\left(2 h\left(C \lambda^{\frac{1}{p-1}}\right)\right)^{\frac{1}{q-1}} \lambda^{\frac{1}{q-1}} \mu_{q}^{\prime}\right)\right]}{C^{p-1}} \leq 1
$$

then

$$
\begin{aligned}
\int\left|\nabla z_{1}\right|^{p-2} \nabla z_{1} \nabla w \mathrm{~d} x & =\lambda\left(\frac{C}{\mu_{p}^{\prime}}\right)^{p-1} \int w \mathrm{~d} x \\
& \geq \lambda \int\left[\|g\|_{\infty} a\left(C \lambda^{\frac{1}{p-1}}\right)+f\left(\left(2 h\left(C \lambda^{\frac{1}{p-1}}\right)\right)^{\frac{1}{q-1}} \lambda^{\frac{1}{q-1}} \mu_{q}^{\prime}\right)\right] \mathrm{d} x \\
& \geq \lambda \int\left[g(x) a\left(C \lambda^{\frac{1}{p-1}} \frac{\kappa_{p}}{\mu_{p}^{\prime}}\right)+f\left(\left(2 h\left(C \lambda^{\frac{1}{p-1}}\right)\right)^{\frac{1}{q-1}} \lambda^{\frac{1}{q-1}} \kappa_{q}\right)\right] \mathrm{d} x \\
& =\int\left[g(x) a\left(z_{1}\right)+f\left(z_{2}\right)\right] w \mathrm{~d} x
\end{aligned}
$$

Similarly, choosing $C$ large so that

$$
\frac{\|g\|_{\infty}\left(b\left(2 h\left(C \lambda^{\frac{1}{p-1}}\right)\right)^{\frac{1}{q-1}} \lambda^{\frac{1}{q-1}} \mu_{q}^{\prime}\right)}{h\left(C \lambda^{\frac{1}{p-1}}\right)} \leq 1
$$


then

$$
\begin{aligned}
\int\left|\nabla z_{2}\right|^{q-2} \nabla z_{2} \nabla w \mathrm{~d} x & =2 \lambda h\left(C \lambda^{\frac{1}{p-1}}\right) \int w \mathrm{~d} x \\
& \geq \lambda \int\left[\|g\|_{\infty} b\left(z_{2}\right)+h\left(z_{1}\right)\right] w \mathrm{~d} x .
\end{aligned}
$$

Hence by Lemma (1.1), there exist a positive solution $(u, v)$ of $(1)$ such that $\left(\psi_{1}, \psi_{2}\right)$ $\leq(u, v) \leq\left(z_{1}, z_{2}\right)$.

\section{Author details}

${ }^{1}$ Department of Mathematics, Science and Research Branch, Islamic Azad University, Tehran, Iran ${ }^{2}$ Department of Mathematics, Faculty of Mathematical Sciences University of Mazandaran, Babolsar, Iran

\section{Authors' contributions}

SH has presented the main purpose of the article and has used GAA contribution due to reaching to conclusions. All authors read and approved the final manuscript.

\section{Competing interests}

The authors declare that they have no competing interests.

Received: 13 August 2011 Accepted: 2 December 2011 Published: 2 December 2011

\section{References}

1. Ali, J, Shivaji, R: Existence results for classes of Laplacian system with sign-changing weight. Appl Math Anal. 20, 558-562 (2007)

2. Rasouli, SH, Halimi, Z, Mashhadban, Z: A remark on the existence of positive weak solution for a class of (p, q)-Laplacian nonlinear system with sign-changing weight. Nonlinear Anal. 73, 385-389 (2010). doi:10.1016/..na.2010.03.027

3. Ali, J, Shivaji, R: Positive solutions for a class of (p)-Laplacian systems with multiple parameters. J Math Anal Appl. 335 , 1013-1019 (2007). doi:10.1016/j.jmaa.2007.01.067

4. Hai, DD, Shivaji, R: An existence results on positive solutions for class of semilinear elliptic systems. Proc Roy Soc Edinb A. 134,137-141 (2004). doi:10.1017/S0308210500003115

5. Hai, DD, Shivaji, R: An Existence results on positive solutions for class of p-Laplacian systems. Nonlinear Anal. 56, 1007-1010 (2004). doi:10.1016/j.na.2003.10.024

6. Canada, A, Drabek, P, Azorero, PL, Peral, I: Existence and multiplicity results for some nonlinear elliptic equations. A survey Rend Mat Appl. 20, 167-198 (2000)

doi:10.1186/1687-2770-2011-52

Cite this article as: Haghaieghi and Afrouzi: Sub-super solutions for $(p-q)$ Laplacian systems. Boundary Value Problems 2011 2011:52.

\section{Submit your manuscript to a SpringerOpen ${ }^{\circ}$} journal and benefit from:

- Convenient online submission

- Rigorous peer review

- Immediate publication on acceptance

- Open access: articles freely available online

- High visibility within the field

- Retaining the copyright to your article

Submit your next manuscript at $\boldsymbol{s p r i n g e r o p e n . c o m ~}$ 